\title{
NUMERICAL STUDY OF ENHANCING HEAT TRANSFER USING TWISTED TAPE INSERTS
}

\author{
A. Khalil, E.A. Elshenawy, Y.A.F. El-Samadony, and A.M. Salem \\ Mech. Power Eng. Dep., Faculty of Eng., Tanta University, Egypt \\ Email:eng73a@yahoo.com
}

\begin{abstract}
In the present work, a numerical study of heat transfer for flow inside tube using twisted tapes inserts is presented. Four models have been studied which are plain tube, tube with single twisted tape, tube with double twisted tapes co-swirling flow and tube with double twisted tapes counter swirling flow. The effect of twisted tape pitch and width ratio on heat transfer, Nusselt number, pressure drop and effectiveness are studied. It is found that Nusselt number, effectiveness and pressure drop are increased as pitch ratio decreased. Also, the results show that counter swirling tape inserts has Nusselt number higher than co-swirling tapes, single tapes, and plain tube by about 5-9 \%, 14-22 \%, and 55-80\% respectively. The results indicate also that twisted tapes with clearance ratio are less effective compared to the types of zero clearance (tight fit tapes). Finally, a good agreement has been found between the present numerical results and published experimental results.
\end{abstract}

Keywords: Heat transfer; Twisted tapes; Pitch ratio.

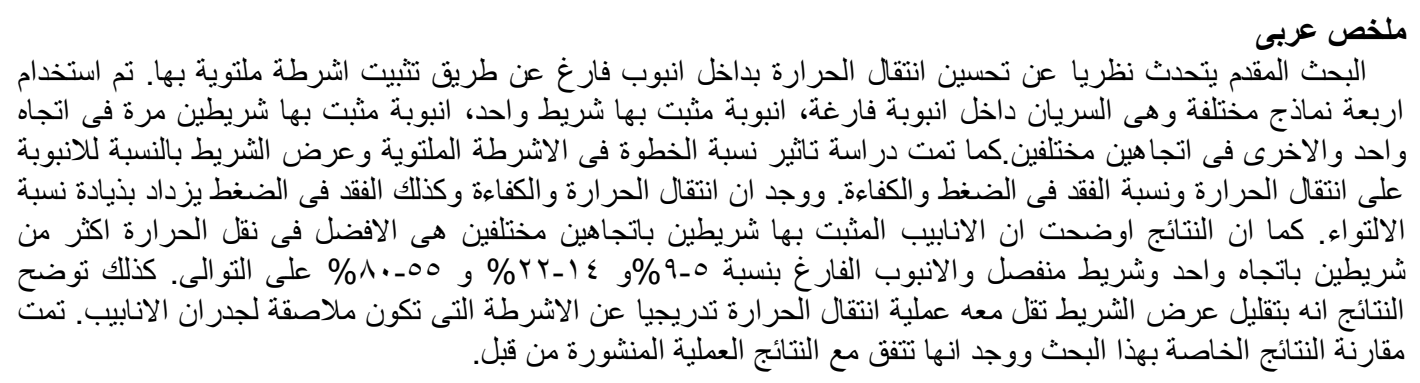

\section{INTRODUCTION}

Heat exchanger is a device that is used to transfer thermal energy between two or more objects at different temperatures. The design procedure of heat exchangers is quite complicated, as it needs exact analysis of heat transfer rate and pressure drop estimations apart from issues such as long-term performance and economic aspects. The major challenge in designing a heat exchanger is to make the equipment compact and achieve a high heat transfer rate using minimum pumping power. To enhance heat transfer, passive, active or a combination of passive and active methods augmentation techniques are used. Passive

techniques, where inserts are used in the flow passage to augment the heat transfer rate, are advantageous compared with active techniques, this is because the insert manufacturing process is simple and these techniques can be easily employed in an existing heat exchanger. The research activities in this sector are still increasing to improve the performance of heat exchangers.

For several years, researchers studied many ways to enhance heat transfer in heat exchangers. After the success of the use of twisted tape for heat transfer augmentation which was early reported by Whitham [1]. Further improvements of thermal 
performance for tubes with various geometries of twisted tape inserts have been released [5 to 7].

Fokeer [2] studied experimentally the swirl flow that were generated by three lobed helical pipe mounted within a laboratory scale pneumatic conveying rig for turbulent flow. He found that heat transfer rate and swirl was increased in this type of tubes. On the other hand it was very expensive method for enhancing heat transfer and the results of heat transfer were lower than those of twisted tapes insertion.

Murugesan [3] studied experimentally heat transfer, friction factor and thermal enhancement factor characteristics of a double pipe heat exchanger Royds[5], Saha et al. [6], and Sarma et al. [7] presented a new approach in predicting the convective heat transfer coefficient in a tube with twisted tape inserts of different pitch to diameter ratios.

Saha et al. [8] and Krishna et al. [9] investigated experimentally heat transfer characteristics of circular tube fitted with straight full twist inserts. Experiments were carried out for turbulent flow using straight full twist insert with spacers and also twisted tapes connected by thin circular rods. Similar trend of increasing Nusselt number with Reynolds number was observed. They found that reducing tape width yields poor results while full length twisted tape is more effective than separated twisted tapes.

Bas and Ozceyhan[10] and Eiamsa-ard et al. [11] investigated experimentally flow friction and heat transfer behavior in a twisted tape swirl generator inserted in a tube. The twisted tapes are inserted separately from the tube wall. Eiamsa et al. [11] used two clearance ratios $(\mathrm{c} / \mathrm{D}=0.0178$ and 0.0357$)$ in the range of Reynolds numbers from 5132 to 24,989 and also with clearance ratio of zero for comparison for three twist ratios. They found that Nusselt number increases with the decrease of twist ratio, clearance ratio and the increase of Reynolds number. Results show that twisted tapes with clearance ratio are less effective compared to the types of zero clearance (tight fit tapes).

Bhattacharyya et al. [12] studied experimentally friction factor and Nusselt number data for laminar flow through a circular duct having integral transverse ribs and fitted with centre-cleared twisted-tape. The major findings of this experimental investigation are that the centre-cleared twisted tapes in combination with transverse ribs perform significantly better than the individual enhancement technique acting alone for laminar flow through a circular duct up to a fitted with square-cut twisted tapes and plain twisted tapes. He found. Bhuiya [4] studied experimentally the heat transfer performance and friction factor characteristics in a circular tube fitted with twisted wire brush inserts for turbulent flow with four twist wire densities. The results indicate that the presence of twisted wire brush inserts led to a large effect on the enhancement of heat transfer with corresponding increase in friction factor over the plain tube. Nusselt number and friction factor of using the twisted wire brush inserts were found to be increased up to 2.15 and 2.0 times, respectively, than those over the plain tube values.

certain amount of centre-clearance. Saha et al. [13] studied enhancing heat transfer of laminar flow of viscous oil through a circular tube having integral helical rib roughness and fitted with helical screw-tapes. They found that the conventional twisted tapes and helical screwtape are fundamentally similar as far as the flow physics is concerned. Both friction factor and Nusselt number behave similarly.

Sharma et al. [14] estimated heat transfer coefficient and friction factor in the transition flow with low volume concentration of $\mathrm{Al} 2 \mathrm{O} 3$ nanofluid flowing in a circular tube and with twisted tape insert. The heat transfer coefficient of nanofluid flowing in a tube with $0.1 \%$ volume concentration is $13.77 \%$ at 3000 Reynolds number and $23.7 \%$ higher when compared with water at Reynolds number of 9000. They also made a comparison between their work and previous works which show a good agreement for their results.

Eiamsa-ard et al. [15] studied experimentally the influences of twin-counter/co-twisted tapes on heat transfer rate, friction factor, and thermal enhancement index. The tests were conducted using the counter tapes and co-swirl tapes with four different twist ratios $(\mathrm{y} / \mathrm{w}=$ 2.5, 3.0, 3.5 and 4.0) for Reynolds numbers range between 3700 and 21,000 under uniform heat flux conditions. They found that heat transfer rates in the tube fitted with the counter tapes are around $12.5-44.5 \%$ and $17.8-50 \%$ higher than those with the co-swirl tapes and single tapes inserts. In addition, empirical correlations of the heat transfer, friction factor, and thermal enhancement index were also reported. Chang et al. [16] carried out heat transfer and pressure drop measurements in three test tubes fitted with single, twin, and triple twisted-tapes with Reynolds number (Re) varying from 1,500 to 14,000 . The heat transfer results show a gradual increase for triple twisted tapes inserts. The local Nusselt numbers in the tubes fitted with single, twin, 
and triple twisted tapes were, respectively, 1.5-2.3, 1.98-2.8, and 2.86-3.76 times of the Dittus-Boelter levels. On the other hand the friction factor increases significantly with the insert of tapes.

Manglik and Bergles [17] considered all these effects and developed laminar flow correlations for the friction factor and Nusselt number including the swirl parameter, which defines the interaction between viscous, convective inertia and centrifugal forces. These correlations pertain to the constant wall temperature case for fully developed flow, based on both previous data and their own experimental data. They also developed correlations for turbulent flow. For an isothermal friction factor, the correlation describes most available data for laminar, transitional and turbulent flows within 10 per cent deviation. A literature of over hundred papers of heat transfer enhancement using twisted tapes was described by Dewan et al. [18].

In the present study a detailed numerical work describing the effect of inserting single, double co-swirling and double counter swirling insert tapes in a plain circular tube with zero clearance ratio and with different clearance ratios. The tapes were used with many pitch lengths to get the optimum design.

\section{NUMERICAL WORK}

\subsection{Geometry and Physical definition}

The present work simulates four steadystate simulation cases which are flow inside plain tube, tube with single twisted tape (Figure 1), which shows apart from Eiamsaard[15] experiment, tube with double twisted tapes co-swirling flow (Figure 2) and tube with double twisted tapes counter swirling flow (figure 3). The tube dimensions are $2 \mathrm{~cm}$ in diameter and $1 \mathrm{~m}$ in length. A twisted tape with different pitch ratios, $\mathrm{G}=\mathrm{y} / \mathrm{d}$, and width ratio, $\mathrm{Z}=\mathrm{W} / \mathrm{d}$, was inserted into the tube. The geometry was assumed as a 3-D region or domain with a 2-D inlet (cold water at $25^{\circ} \mathrm{C}$ ) and a 2-D outlet. The tapes material is aluminum and has $1 \mathrm{~mm}$ thickness. The outside surface of the tube was assumed to be in isothermal condition at $100{ }^{\circ} \mathrm{C}$. The instantaneous equations of mass, momentum and energy conservation can be written as follows in a stationary frame:

2.1.1. The Continuity Equation $\partial p / \partial \mathrm{t}+\nabla \cdot(p \mathrm{U})=0$

\subsubsection{The Momentum Equations}

$$
\frac{\partial(p \mathrm{U})}{\partial \mathrm{t}}+\nabla \cdot(p \mathrm{U} \times \mathrm{U})=-\nabla \mathrm{P}+\nabla \cdot \tau+\mathrm{S}_{\mathrm{M}}
$$

Where the stress tensor, $\tau$, is related to the strain rate by

$$
\tau=\mu\left(\nabla U+(\nabla U)^{T}-\frac{2}{a} \delta \nabla \cdot U\right)
$$

2.1.3. The Total Energy Equation

$$
\begin{aligned}
& \frac{\partial\left(\mathrm{ph}_{\mathrm{tot}}\right)}{\partial \mathrm{x}}-\frac{\partial \mathrm{p}}{\partial \mathrm{t}}+\nabla \cdot\left(\rho \mathrm{U} \mathrm{h}_{\mathrm{tot}}\right)=\nabla \cdot(\lambda \nabla \mathrm{T})+ \\
& \nabla \cdot(\mathrm{U} \cdot \tau)+\mathrm{U} \cdot \mathrm{S}_{\mathrm{MI}}+\mathrm{S}_{\mathrm{E}}
\end{aligned}
$$

Where $h_{\text {tot }}$ is the total enthalpy, related to the static enthalpy $h\left(\mathrm{~T}_{w} \mathrm{P}\right)$ by:

$\mathrm{h}_{\text {tot }}=\mathrm{h}+\frac{1}{2} \mathrm{U}^{2}$

The term $\nabla \cdot(\mathrm{U} \cdot \tau)$ represents the work due to viscous stresses and is called the viscous work term.

The term $\mathrm{U} \cdot \mathrm{S}_{\mathrm{M}}$ represents the work due to external momentum sources and is currently neglected.

\section{2: HEAT TRANSFER CALCULATION (NU)}

The heat transfer rate at steady state is assumed to be equal to the heat loss from the test section which may be expressed as:

$\mathrm{Q}_{\text {water }}=\mathrm{Q}_{\text {conv }}$

As $Q$ is the heat transfer rate

Then the heat transferred from the water flow can be drawn as,

$\mathrm{Q}_{\text {wate }} \mathrm{r}=\mathrm{m} \cdot \mathrm{C}_{\mathrm{P}}\left(\mathrm{T}_{\text {out_ }} \mathrm{T}_{\text {in }}\right)$

The heat transferred from the tube wall by convection may be also written as,

$\mathrm{Q}_{\text {conv }}=\mathrm{hA} \Delta \mathrm{T}_{\mathrm{LMTD}}$

Where $\triangle T L M T D$ is the logarithmic mean temperature difference and is measured as following

$\Delta \mathrm{T}_{\mathrm{LMTD}}=\frac{\text { Tout-Tin }}{\mathrm{Ln} \frac{\mathrm{TW}-\mathrm{Tin}}{\mathrm{TW}-\mathrm{Tout}}}$

and $\mathrm{A}$ is the total surface area of the inside tube wall, which can be expressed as: $A=\pi \mathrm{DL}$, where $\mathrm{D}$ is the inner diameter of the test tube. The average heat transfer coefficient $(h)$ for the test tube can be calculated by the combination of Eqs. (2) and (3), and from the results, the average Nusselt number is defined as follow, $\mathrm{Nu}=\mathrm{hDh} / \mathrm{k} \quad(10)$

Where $\mathrm{K}$ is the local thermal conductivity of the fluid which calculated from the fluid properties at the local mean bulk fluid temperature $\left(\mathrm{T}_{\mathrm{b}}=\left(\mathrm{T}_{\text {out }}+\mathrm{T}_{\text {in }}\right) / 2\right)$. And $\mathrm{D}_{\mathrm{h}}$ is the hydraulic diameter of the flow path. Nusselt number, Prandtl number, Reynolds number, and all of thermo-physical properties of the fluid were calculated of the basis of water properties corresponding to the bulk fluid temperature $(\mathrm{Tb})$. The Reynolds number based on the total flow rate at the inlet of the test section is expressed as: 
$\operatorname{Re}=\rho \mathrm{VD}_{\mathrm{h}} / \mu$

And effectiveness can be calculated from $\varepsilon=\frac{\text { MCp(Tout_Tin })}{\text { MCp }(\text { Tmax_Tin })}=\frac{Q}{Q \max }$

It is very important to validate the present plain tube data of Nusselt number in fully developed straight/axial flow with the correlations from the previous studies.

A computational fluid dynamic computer program "CFX" was used to show the effect of each parameter individually. The convergence was achieved after much iteration. The following results could be obtained; output temperature, pressure drop, velocity distribution, and heat transfer coefficient. Also thermal enhancement index $(\varphi)$ was calculated. It is defined as the ratio of Nusselt number in a tube provided by any type of twisted tape inserts to Nusselt number in a plain tube. The enhancement index for plain tube was considered to be unity for the range of Reynolds number studied (1000 to 20000). The clearance ratio $(\mathrm{C})$ as the ratio between the clearance between the tape and inner wall of the tube to the tape width.

\section{RESULTS AND DISCUSSION}

A detailed study of the factors affecting heat transfer coefficient and pressure drop was made in order to obtain the optimum design of tapes. This study included the effect of inlet flow Reynolds number, pitch ratio and width ratio for plain tube, tube with single twisted tape, and tube with double twisted tapes coswirling flow as well as tube with double twisted tapes counter swirling flow.

\subsection{PLAIN TUBE}

Figure (4) illustrates the variation of Nusselt number with Reynolds number for a plain tube. As expected, it can be seen from this figure that as Reynolds numbers increases Nusselt number significantly increases. Also, it can be noticed from Figure (4) that the results of the present model have a good agreement with Dittus and Boelter [19] heat transfer correlation inside a plain tube $\left(\mathrm{Nu}=0.023 \operatorname{Re}^{0.80} \operatorname{Pr}{ }^{0.40}\right)$ and also with the results of Sharma [14]. Comparing both results we have a mean percentage deviation in Nusselt number with the current work of about (3-5) $\%$.

\subsection{EFFECT OF SINGLE TWISTED TAPE PITCH RATIO}

Figure (5) represents the variation of Nusselt number with Reynolds number for plain tube and for tube with inserted single twisted tape at different pitch ratios. It can be seen from this figure that tube with inserted single twisted tape has Nusselt number higher by about (34-47) \% than plain tube case. This is because insertion of twisted tape decreases the hydrodynamic and thermal boundary layer thickness as well as the tube hydraulic diameter. In addition, by inserting the twisted tape; the total fluid residence time inside the tube due to longer traveling distance and fluid swirl intensity will be increased. All of the previous factors cause heat transfer rate to be increased. Also, it can be seen from Figure (5) that as pitch ratio decreases, Nusselt number increases especially at high Reynolds numbers. This is because as pitch ratio decreases fluid pressure drop significantly increases as shown in Figure (6). This causes fluid flow velocity to be decreased and consequently Nusselt number will be decreased. Therefore, the resultant average Nusselt number will insignificantly increase. The velocity contour through the tube length is shown in figure (20A).

As seen in Figure (6), there is a considerable increase in pressure drop with Reynolds number for all twist ratios used for single tape inserts. It is noticed that increasing Reynolds number and decreasing pitch ratio increasing the pressure drop, which has consequently results on the pumping power. It was found that single tapes inserts increases pressure drop by (45-80) \% compared with plain tube case.

\subsection{EFFECT OF CO-SWIRLING TAPES PITCH RATIO}

Figure (7) illustrates the variation of Nusselt number with Reynolds number for tubes with inserted co-swirling twisted tapes at different pitch ratios. It can be seen from this figure that tube with inserted co-swirling twisted tapes has Nusselt number higher by about (45-65) \% than plain tube case and generally higher than the single tapes by $12 \%$. The figure shows a little increase in heat transfer between various types of co-swirling tapes inserts, and indicates also that all inserts of co-swirling tapes have higher heat transfer rate and Nusselt number than the single tape one.

It can be seen that with decreasing pitch ratio, heat transfer and Nusselt number increase. There is a large increase in pressure drop as shown in figure (8) by about (105-155) $\%$ over that of plain tube. Pressure drop increases with increasing Reynolds number and with decreasing pitch ratio. This is because of the repeated action of strong intensity of vortex in the tube with co-swirling tapes that have more effects in the counter flow tapes. Inserting two parallel tapes enhances fluid 
mixing and generates higher swirl flow which appears in Figure (20B) where recirculation zones appear on the top/bottom of vortex core and the generated swirl flows show just small contact zone in the clearance between two twisted tapes, indicating little interaction between them. Therefore, the mixing in the tube equipped with the co-swirl tapes is dominantly enhanced by rather independent one swirl flows.

\section{4: EFFECT of COUNTER SWIRLING TAPES PITCH RATIO}

Figure (9) illustrates the variation of Nusselt number with Reynolds number for tube with inserted counter swirling twisted tape inserts at different pitch ratios. It can be seen from this figure that tube with inserted counter swirling twisted tapes has Nusselt number higher than co-swirling tapes, single tapes, and plain tube by (5-9) \%, (14-22) \%, and (55-80) \% respectively. The turbulence intensity and swirl flow here is more than the plain tube, the single tape cases, or even the co-swirling flow cases. In general, inserting tapes in the flow passage to augment the heat transfer rate reduces the hydraulic diameter of the flow passage. Heat transfer enhancement is mainly due to flow blockage, partitioning of the flow and secondary flow. Flow blockage increases the pressure drop and leads to increased viscous effects because of a reduced free flow area. Blockage also increases the flow velocity and in some situations leads to a significant secondary flow. Secondary flow further provides a better thermal contact between the surface and the fluid because secondary flow creates swirl and the resulting mixing of fluid improves the temperature gradient, which ultimately leads to a high heat transfer coefficients.

On the other hand, we can easily notice the gradual increase in pressure drop and hence the friction factor which lead to increase the pumping power used in these cases. Figure(9) shows a little increase in heat transfer between various types of counter swirling inserts, and also indicates that all inserts of counter swirling tapes have higher heat transfer rate and Nusselt number than the co-swirling and single tape inserts. Counter inserts have higher heat transfer rate by (55-80) \% over that of plain tube case. Here we have the most effective case in our study. We have the highest Nusselt number of about 290 with the lowest pitch ratio of 2.5 where the temperature difference between exit and inlet is about $31 \mathrm{~K}$.

Counter swirl inserts provide better temperature distribution than the other inserts.
This is attributed to more efficient mixing inside the tube fitted with the counter tapes which is influenced by a stronger swirl flow.

Figure (10) shows the gradual increase of pressure drop for counter tapes inserts for all twist ratios used. It is found that pressure drop in this type of insertion is about (105-170)\% over than plain tube case.

The swirls generated by twin counter twisted tapes are found to be converged in the clearance between two twisted tapes; this apparently provides higher intensity of vortex stronger than that generated by the co-swirling tapes as shown in Figure (18). In addition, the recirculation zone is not induced in the tube with the counter-flow tapes as the strong swirl flows impinge to tube wall. Therefore, it can be addressed here in that the higher interaction between swirl flows (or high vortex strength) induced by the counter tapes leads to superior fluid mixing, resulting in uniform fluid temperature in tubes, and thus more efficient heat transfer in comparison with the co-swirl tapes.

\section{COMPARISON BETWEEN SINGLE AND DOUPLE TWISTED TAPES}

Figure (11) illustrates the variation of Nusselt number with Reynolds number for plain tube, single twisted tape, double twisted tapes co-swirling flow and double twisted tapes counter swirling flow for the most effective pitch ratio $(\mathrm{G}=2.5)$. The results show that counter swirling tape inserts have Nusselt number higher than co-swirling tapes, single tapes, and plain tube by (5-9) \%, (14-22) \%, and $(55-80) \%$ respectively.

Figure (12) represents the variation of heat exchanger effectiveness with Reynolds number for plain tube and for tube with inserted single, double co-swirling, and double counter twisted tape at the smallest pitch ratio in our work $(\mathrm{G}=2.5)$. It can be seen from this figure that counter tapes inserts have higher effectiveness by $91 \%$ than plain tube case. And co-swirling flow inserts have higher effectiveness by $81 \%$ than plain tube case. While single tape inserts have higher effectiveness by $65 \%$ than plain tube case. Counter inserts have effectiveness higher than co-swirling tapes, single tapes, and plain tube by $8 \%, 14 \%$, and $100 \%$ respectively for pitch ratio $(\mathrm{G}=2.5)$.

\section{ENHANCEMENT INDEX.}

Figure (13) shows the thermal enhancement index for all cases investigated in the present work which is considered to be unity for plain tube under the range of Reynolds number studied. Thermal enhancement index is very high for counter 
inserts as shown in figure (13). It is high in the laminar flow regions. As we stated earlier that in the turbulent flow, pressure drop increases significantly which affects the increase of heat transfer; the reason why it is not increasing significantly like laminar flow regions. From CFX post we have figures indicating velocity distribution like Figure(20A) which shows the path line flow through a tube fitted with single tape insert where we can see the mix very well in (side view) which leads to enhancing the heat transfer process. This will be shown further more in co-swirling flow and counter swirl type inserts. The streamlines for the flow passage through co-swirl tapes inserts are shown. Each streamline is induced along each side of tape with axisymmetric profile in direction led by the tape geometry. With the close look for twin co-twisted tapes recirculation zones appear on the top/bottom of vortex core and the generated swirl flows show just small contact zone in the clearance between two twisted tapes, indicating little interaction between them as shown in figure (20B). Therefore, the mixing in the tube equipped with the co-swirling tapes is dominantly enhanced by rather independent swirl flows.

\section{EFFECT OF VARYING TAPE WIDTH}

Changing the tape width in the present work, we found that heat transfer falls dramatically as we decrease the clearance ratio. Studying this effect in the three inserts used; single, double co-swirling, and double counter swirling tapes for different pitch ratios we have the following results:

Three clearance ratios are used $(\mathrm{C}=0$, $\mathrm{C}=0.024$, and $\mathrm{C}=0.036$ ). It is found that for the three ratios single tapes inserts with zero clearance have higher heat transfer rate than $\mathrm{C}=0.024$ and $\mathrm{C}=0.036$ by $12.3 \%$ and $16 \%$ respectively as shown in Figure (14). This figure shows the variation of Nusselt number with Reynolds number for single tape insert at different clearance ratios for a fixed twist ratio $\mathrm{G}=2.5$.

For co-swirling tapes using pitch ratio of 3.5 and by using the same clearance ratios mentioned above. The results show that for the three ratios co-swirling tape inserts with zero clearance have higher heat transfer rate than $\mathrm{C}=0.024$ and $\mathrm{C}=0.036$ by $13.7 \%$ and $20.2 \%$ respectively as shown in Figure (15). This figure shows the variation of Nusselt number with Reynolds number for co-swirling tape inserts at different clearance ratios for a fixed twist ratio $\mathrm{G}=3.5$.
For counter swirling tapes using pitch ratio of 5 and by using the same clearance ratios mentioned above. The results show that for the three ratios counter swirling tape inserts with zero clearance have higher heat transfer rate than $\mathrm{C}=0.024$ and $\mathrm{C}=0.036$ by $18.2 \%$ and $24.2 \%$ respectively as shown in Figure (16). This figure shows the relation between Reynolds number and Nusselt number for coswirling tape inserts at different clearance ratios for a fixed twist ratio $\mathrm{G}=5$.

It is noteworthy that the loose tape with smaller clearance ratio gives higher velocity of the fluid flow through the free spacing compared to those with larger clearance ratios due to a smaller free spacing area between the tape and tube wall.

\section{NUMERICAL WORK VERIFICATIONS}

In Figure (17), a comparison between the results of present work and a previous work studied by Manglik\&Bregles[17] under the same conditions for single twisted tape inserts. The present results show a good agreement with their relations within $\pm 4 \%$

Figure (18) compares between the present work results and Eiamsa-ard et al.[15] for double twisted tapes co-swirling flow at different pitch ratios. Also, Figure (19) compares between the present work results and Eiamsa-ard et al.[15] for double twisted tapes counter swirling flow at different pitch ratios. It could be seen from these figures that the previous experimental results are fairly close to the present numerical data.

\section{VELOCITY CONTOURS}

Contour plots of streamlines through the tube with single and double co-swirling twisted tape inserts are respectively displayed in Figure 20(A-B). It is clearly seen that the insertion of single tapes induces swirling flow which causes mixing of fluid and hence increases temperature of fluid flowing and consequently increases heat transfer rate. While co-swirling tapes make flow influenced by a stronger swirl flow and also reversed flow that enhance heat transfer rate than single tape inserts.

Figure (21) shows the swirls generated by twin counter twisted tapes which found to be converged in the clearance between two twisted tapes; this apparently provides higher intensity of vortex strength than that generated by the co-swirl tapes. Therefore, it can be addressed herein that the higher interaction between swirl flows (or high vortex strength) induced by the counter-flow tapes leads to superior fluid mixing, resulting in uniform fluid temperature in tubes, and thus more 
efficient heat transfer in comparison with the co-swirling tapes or single tapes inserts. This confirmed by the obtained results in which, the heat transfer rates in the tube fitted with the counter-swirl flow are noticeably higher than those in the tube fitted with the co-swirl flow for all range Reynolds numbers studied.

\section{CONCLUSIONS}

From this work we have,

1- For general runs we found that heat transfer, Nusselt number and pressure drop increase with decreasing pitch ratio $(\mathrm{G})$ and also with increasing Reynolds number.

2- Counter flow inserts are more efficient than co-swirling inserts or single tapes insert. It was found that counter flow tapes inserts are more effective than co-swirling tapes by nearly (5-9) $\%$ and they are more effective than single tapes by (14-22) \%. Counter tapes are generally more effective than plain tube by $(55-80) \%$.

3- There are increases in heat transfer and Nusselt number in all flow regions but a big increase in heat transfer occurred in laminar flow regions for all inserts.

4- The best swirl ratio for all inserts in the tested range occurs at $\mathrm{G}=2.5$ while in cases of large twist ratios it is not effective.

5- Results show that twisted tapes with clearance ratios more than zero are less effective compared to the types of zero clearance (tight fit tapes).

6- Comparing these results with previous works, it was found deviations of Nusselt number for single tapes insert by nearly $\pm 4 \%$, while for co-swirling and counter swirling tapes by about $\pm 10 \%$. This shows a fairly agreement with previous works.

\section{REFERENCES}

[1] Whitham. J.M., "The effects of retarders in fire tubes of steam boilers" Street Railway Journal 12 (1920).

[2] Fokeer.S., -"An experimental investigation of pneumatic swirl flow induced by a three lobed helical pipe ", International Journal of Heat and Fluid Flow 30 (2009) 369-379

[3] Murugesan.P., "Turbulent Heat Transfer and Pressure Drop in Tube Fitted with Squarecut Twisted Tape" chinese journal of chemical engineering 2010, Vol. 18, 609-617

[4] Bhuiya.M.M.K. , "Heat transfer performance evaluation for turbulent flow through a tube with twisted wire brush inserts", International Communications in Heat and Mass Transfer 39 (2012) 1505-1512

[5] Royds, R. "Heat Transmission by Radiation, Conduction and Convection", 1st edition, 1921, pp. 190-201 (Constable and Camp Limited, London).
[6] Saha, S. K. and Bhunia, K. "Heat transfer and pressure drop characteristics of varying pitch twisted-tape-generated laminar smooth swirl flow". In Proceedings of 4th ISHMTASME Heat and Mass Transfer Conference, India, 2000

[7]Sarma.P.K.,Subramanyam.T.,Kishorea.P.S., Rao.V.D., andKakac.S., "A new method to predict convective heat transfer in a tube with twisted tape inserts for turbulent flow", Int. J. Therm. Sci. 41 (2002) 955-960.

[8] Saha, S. K., Dutta, A. and Dhal, S. K. Friction and heat transfer characteristics of laminar swirl flow through a circular tube fitted with regularly spaced twisted-tape elements. Int. J. Heat and Mass Transfer, 2001, 44, 4211-4223.

[9] Siva Rama Krishna, GovardhanPathipaka, andSivashanmugam" Heat transfer and pressure drop studies in a circular tube fitted with straight full twist" , Experimental Thermal and Fluid Science 33 (2009) 431-438 [10] Halit Bas andVeyselOzceyhan , "Heat transfer enhancement in a tube with twisted tape inserts placed separately from the tube wall", Experimental Thermal and Fluid Science 41 (2012) 51-58

[11] Eiamsa-ard. S. ,Wongcharee b, andSripattanapipat. S., "3-D Numerical simulation of swirling flow and convective heat transfer in a circular tube induced by means of loose-fit twisted tapes", International Communications in Heat and Mass Transfer 36 (2009) 947-955

[12] Suvanjan Bhattacharyya, SubhankarSaha, SujoySaha, "Laminar flow heat transfer enhancement in a circular tube having integral transverse rib roughness and fitted with centrecleared twisted-tape", Experimental Thermal and Fluid Science 44 (2013) 727-735

[13] SubhankarSaha andSujoy Kumar Saha , "Enhancement of heat transfer of laminar flow of viscous oil through a circular tube having integral helical rib roughness and fitted with helical screw-tapes", Experimental Thermal and Fluid Science xxx (2013).

[14] Sharma K.V., SyamSundar ,Sarma. P.K. , -"Estimation of heat transfer coefficient and friction factor in the transition flow with low volume concentration of $\mathrm{Al} 2 \mathrm{O} 3$ nanofluid flowing in a circular tube and with twisted tape insert" International Communications in Heat and Mass Transfer 36 (2009) 503-507

[15] Eiamsa-ard.S. , Thianpong.C ,Eiamsa-ard .P, " Turbulent heat transfer enhancement by counter/co-swirling flow in a tube fitted with twin twisted tapes", Experimental Thermal and Fluid Science 34 (2010) 53-62

[16] Chang. ShyyWoei, Ker-Wei Yu, and Ming Hsin Lu, " Heat transfer in tubes 
fitted with single, twin, and triple twisted tapes" Experimental Heat Transfer, 18, 279294, 2005

[17] Manglik.M, and Bergles. A.E., "Heat transfer and pressure drop correlations for twisted-tape inserts in isothermal tubes: part II" 1983.

[18] Dewan. A., "Review of passive heat transfer augmentation techniques" Journal of Power and Energy 218 (2004) 509-525.

[19] F.P. Incropera, P.D. Witt, T.L. Bergman, and A.S. Lavine,'Fundamentals of Heat and Mass Transfer", John-Wiley \& Sons, 2006.

\section{NOMENCLATURE}

A surface area of cylinder, $\mathrm{m}^{2}$

$\mathrm{C}_{\mathrm{P}}$ specific heat, $\quad \mathrm{Jkg}^{-1} \mathrm{k}^{-1}$

$\mathrm{D}$ tube diameter, $\mathrm{m}$

G twist ratio, $y / \mathrm{W}$

$\mathrm{h}$ heat transfer coefficient, $\mathrm{Wm}^{-2} \mathrm{~K}^{-1}$

$\mathrm{k}$ thermal conductivity of fluid, $\mathrm{Wm}^{-1} \mathrm{~K}^{-1}$

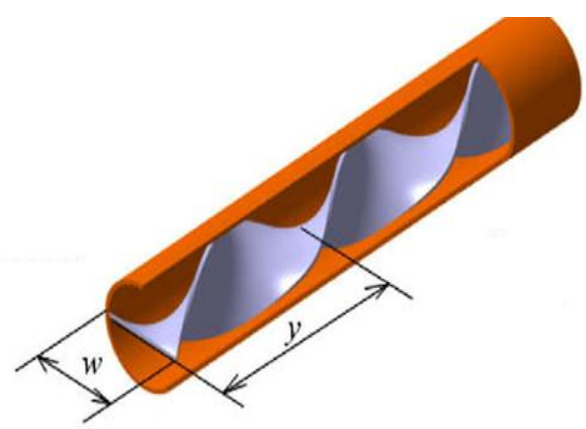

Figure (1)Single tape insert in a plain tube in Eiamsa-ard [15] experiment
$\mathrm{L}$ tube length $\mathrm{m}$

$\mathrm{m}^{\cdot}$ mass flow rate, $\mathrm{kgs}^{-1}$

$\mathrm{Nu}$ Nusselt number, dimensionless

Q Heat transfer rate, W

Re Reynolds number, dimensionless

$\mathrm{T}$ temperature, $\mathrm{K}$

$\Delta \mathrm{T}_{\mathrm{LMTD}}$ logarithmic mean temperature difference

$\mathrm{V}$ velocity, $\mathrm{ms}^{-1}$

w tape width, $\mathrm{m}$

$\mathrm{y} \quad$ pitch length of tape, $\mathrm{mm}$

$\varphi \quad$ Enhancement index

\section{Subscripts}

\begin{tabular}{ll}
\hline $\mathrm{b}$ & bulk \\
In & inlet \\
Out & outlet
\end{tabular}

Greek letters

$\rho \quad$ fluid density, $\mathrm{kgm}^{-3}$

$\varepsilon \quad$ effectiveness, , dimensionless

$\mu \quad$ Fluid dynamic viscosity, $\mathrm{kgs}^{-1} \mathrm{~m}^{-1}$

Figure (2)Co-swirling tapes design.

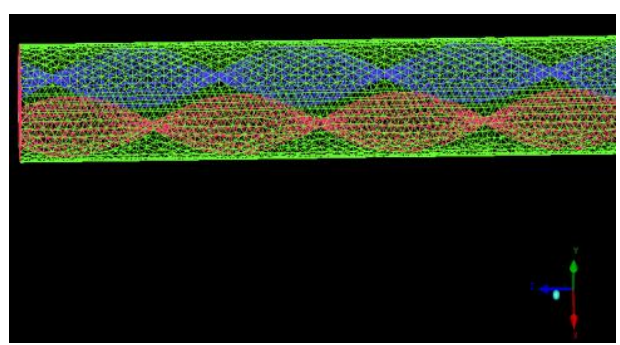

Figure (3): Numerical mesh for counter swirling tapes design.
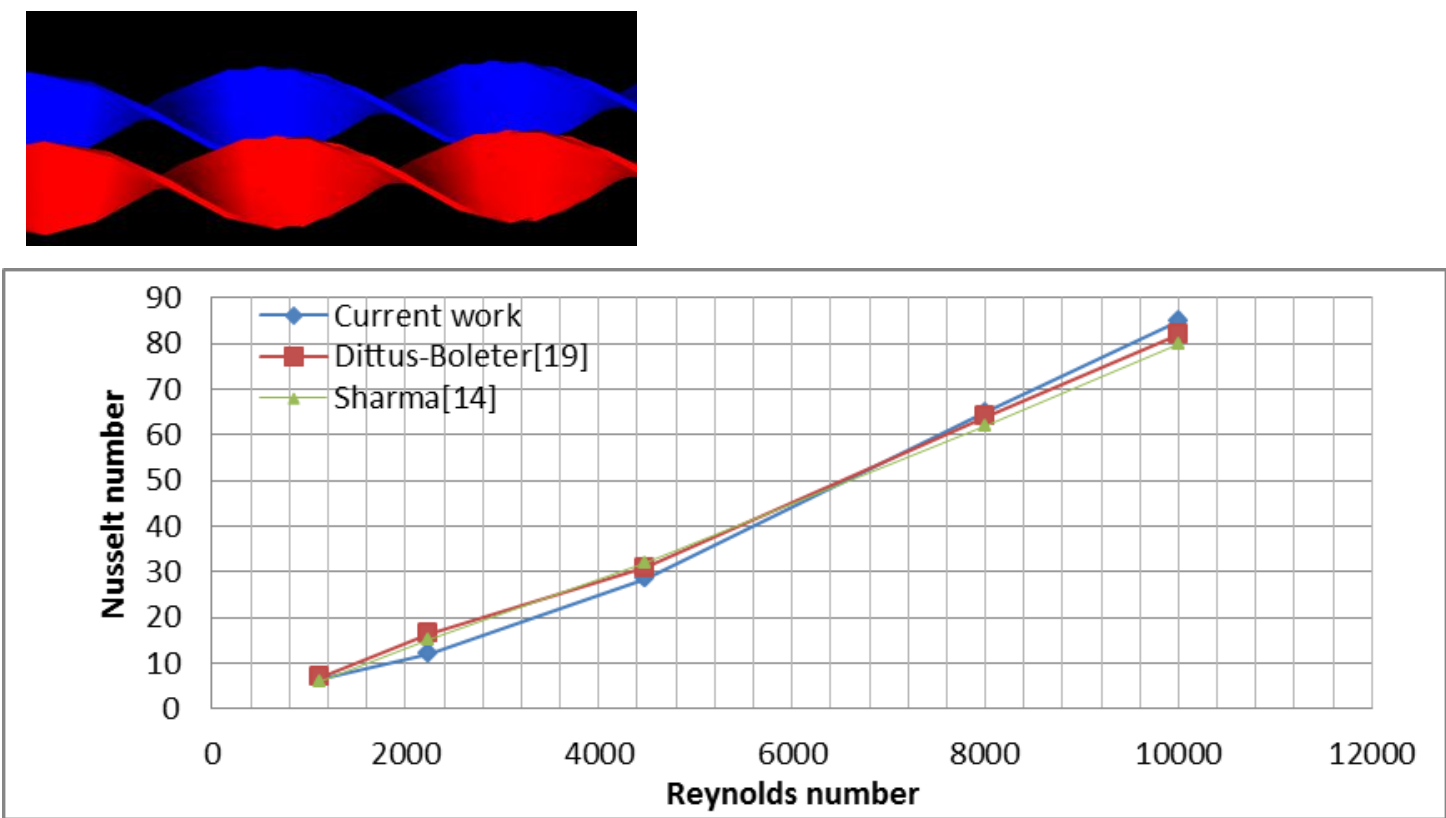

Figure (4):Relation between Reynolds number and Nusselt number for flow inside plain tube 


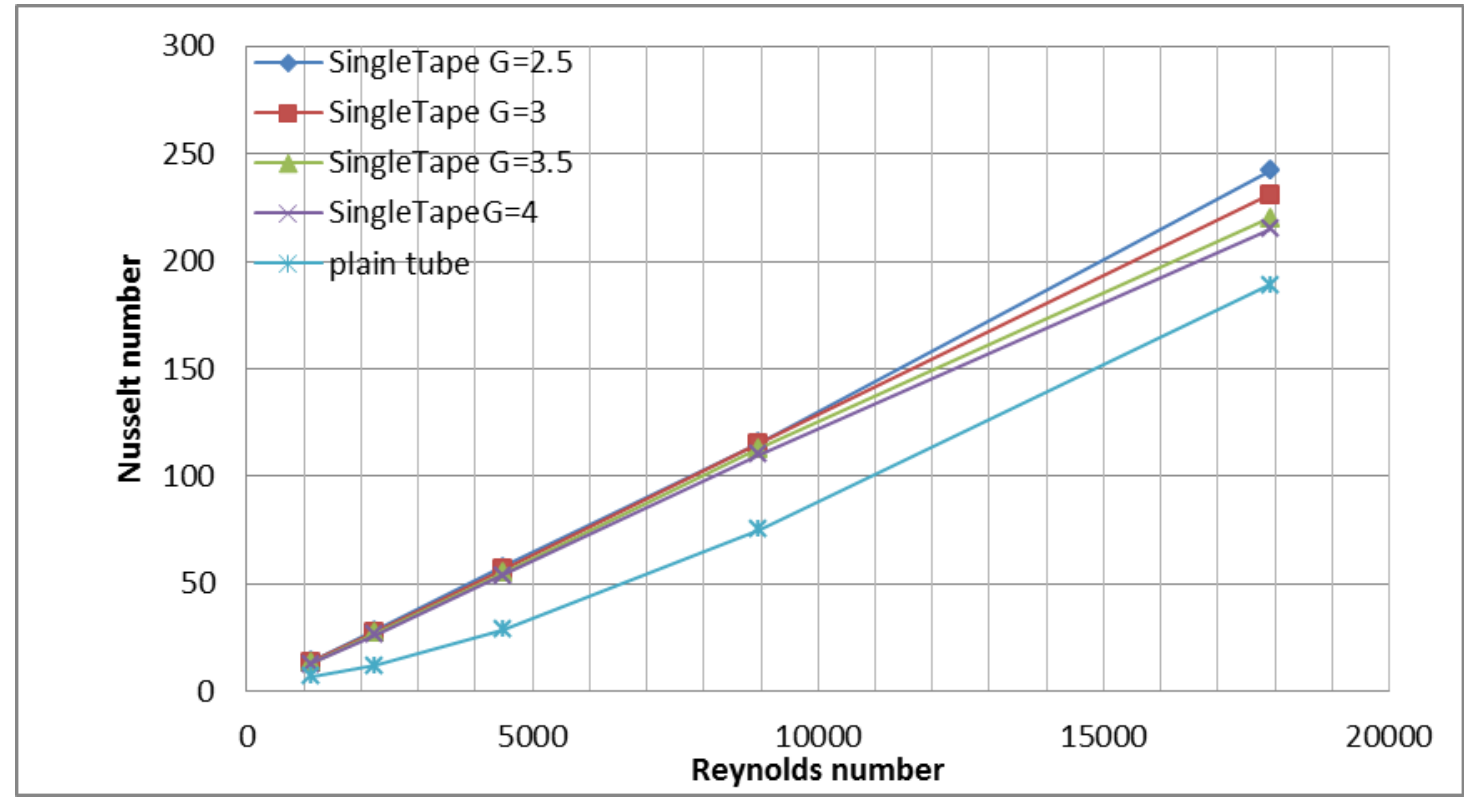

Figure (5): Relation between Reynolds number and Nusselt number for single tapes and plain tube.

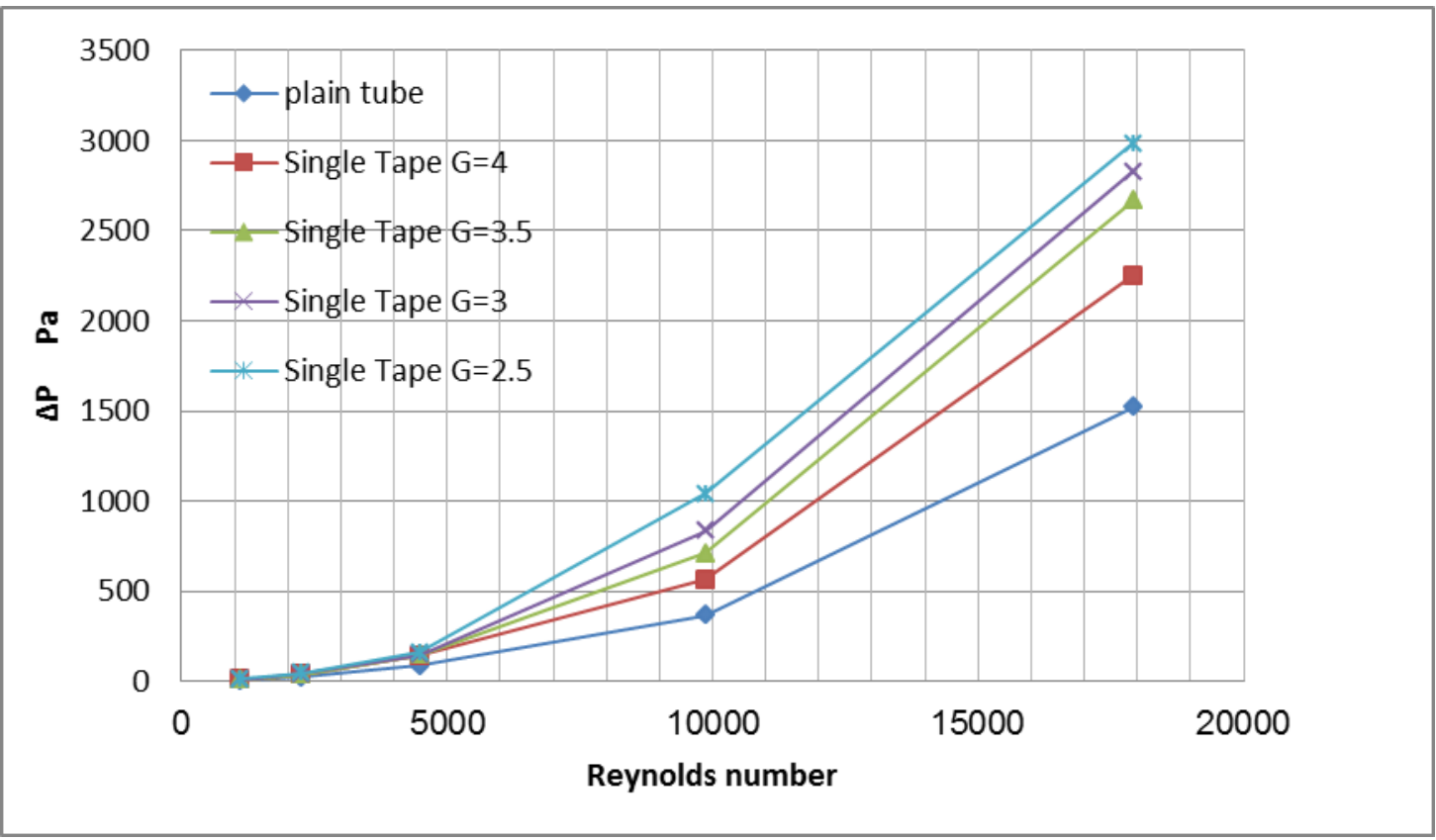

Figure (6):Pressure drop for single tapes inserts and plain tube. 


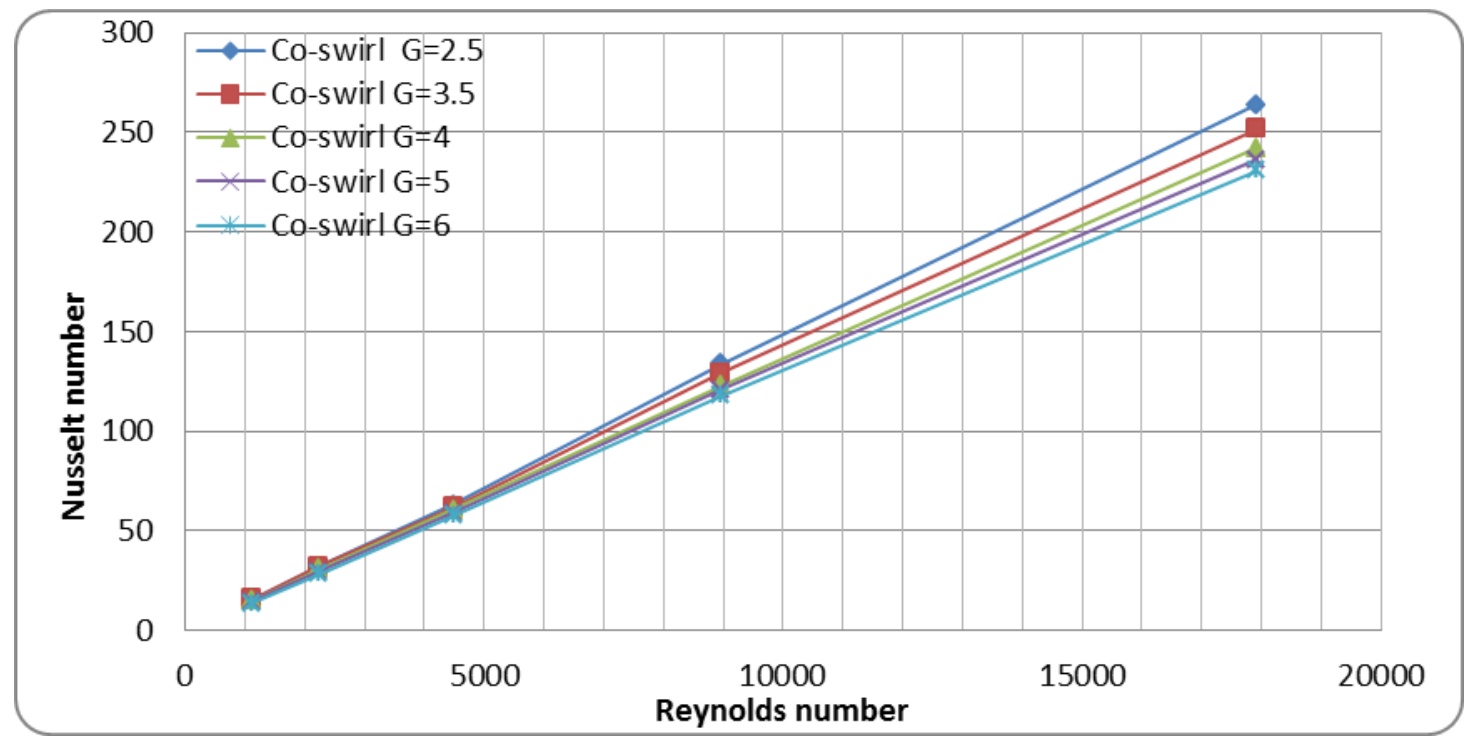

Figure (7): Relation between Reynolds number and Nusselt number for co-swirl tape inserts

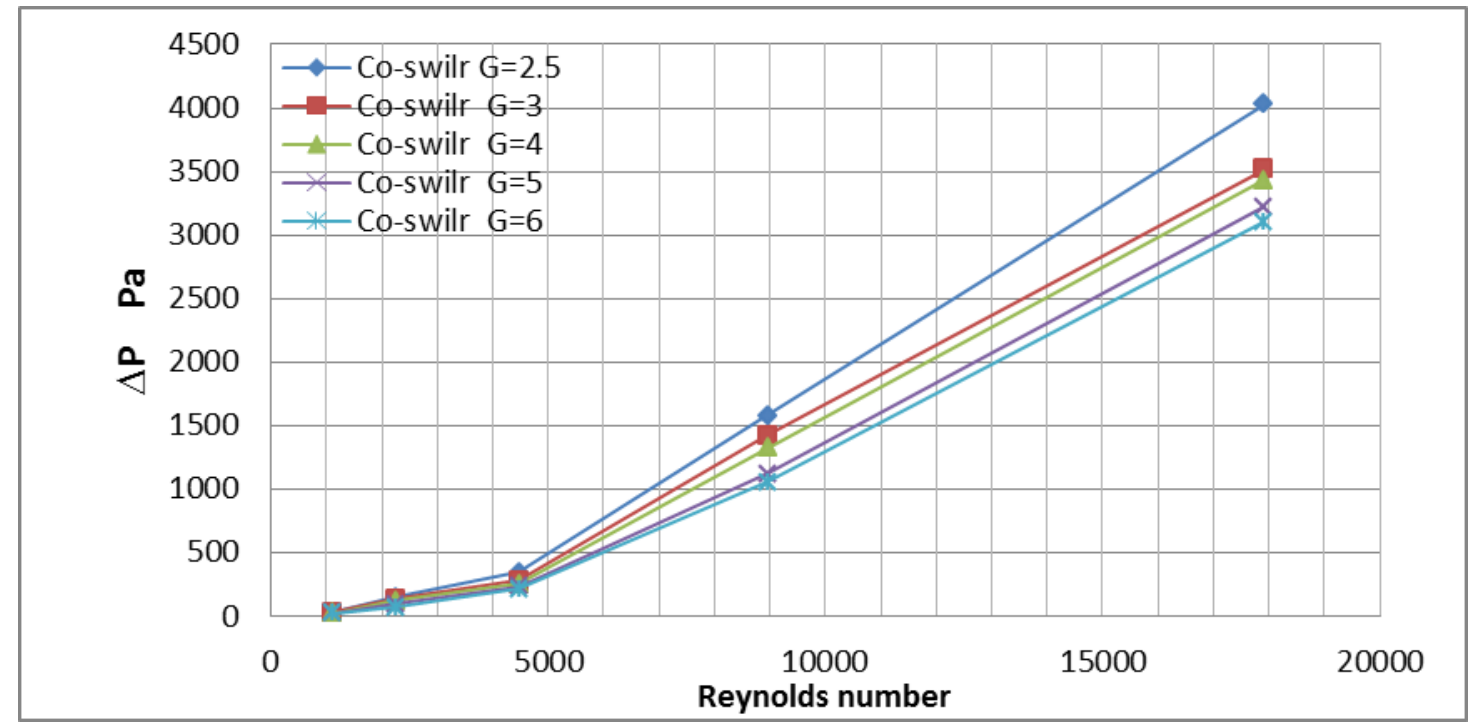

Figure (8): Pressure drop for co-swirling tapes inserts 


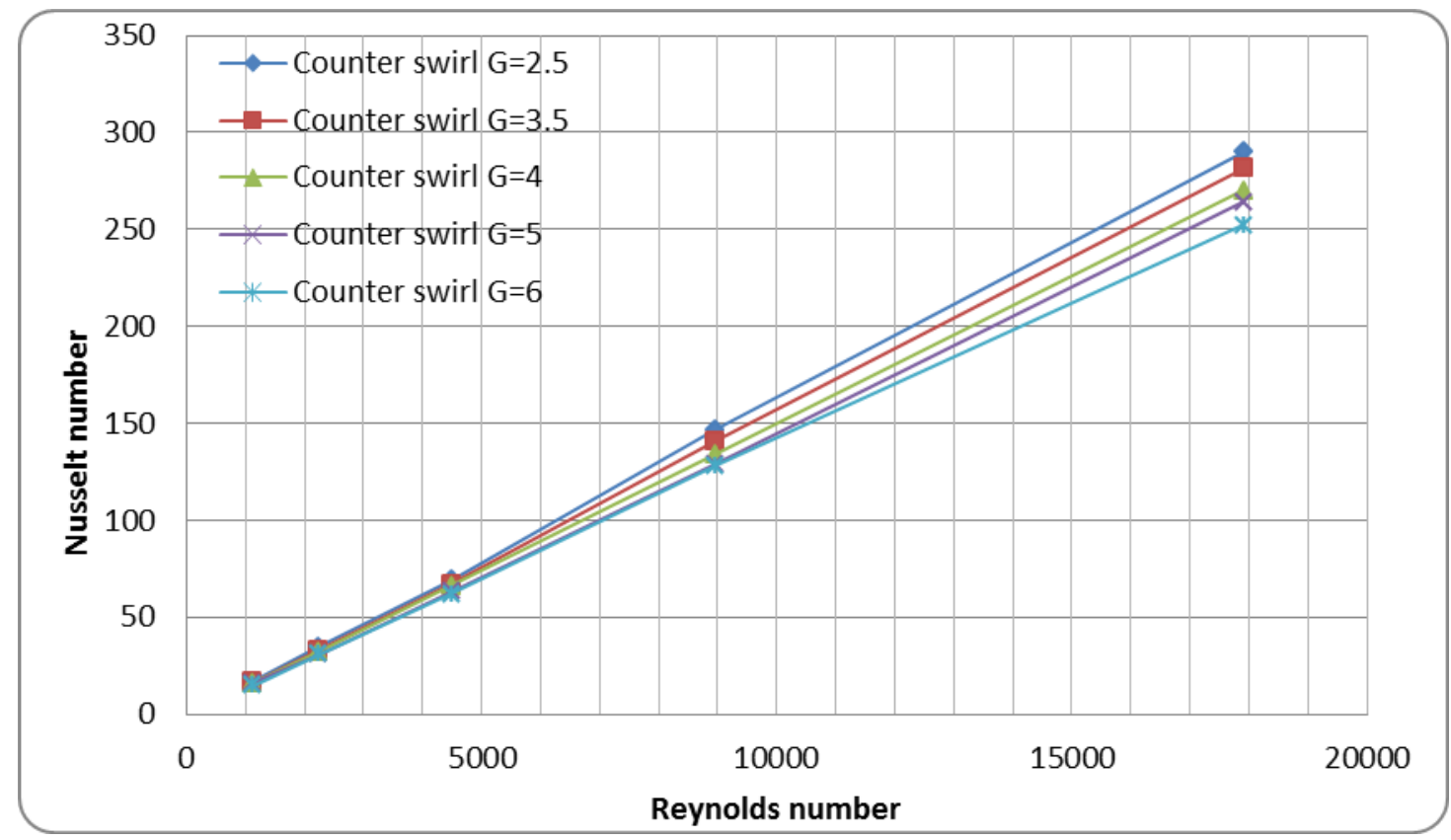

Figure (9): Relation between Reynolds number and Nusselt number for counter tape inserts.

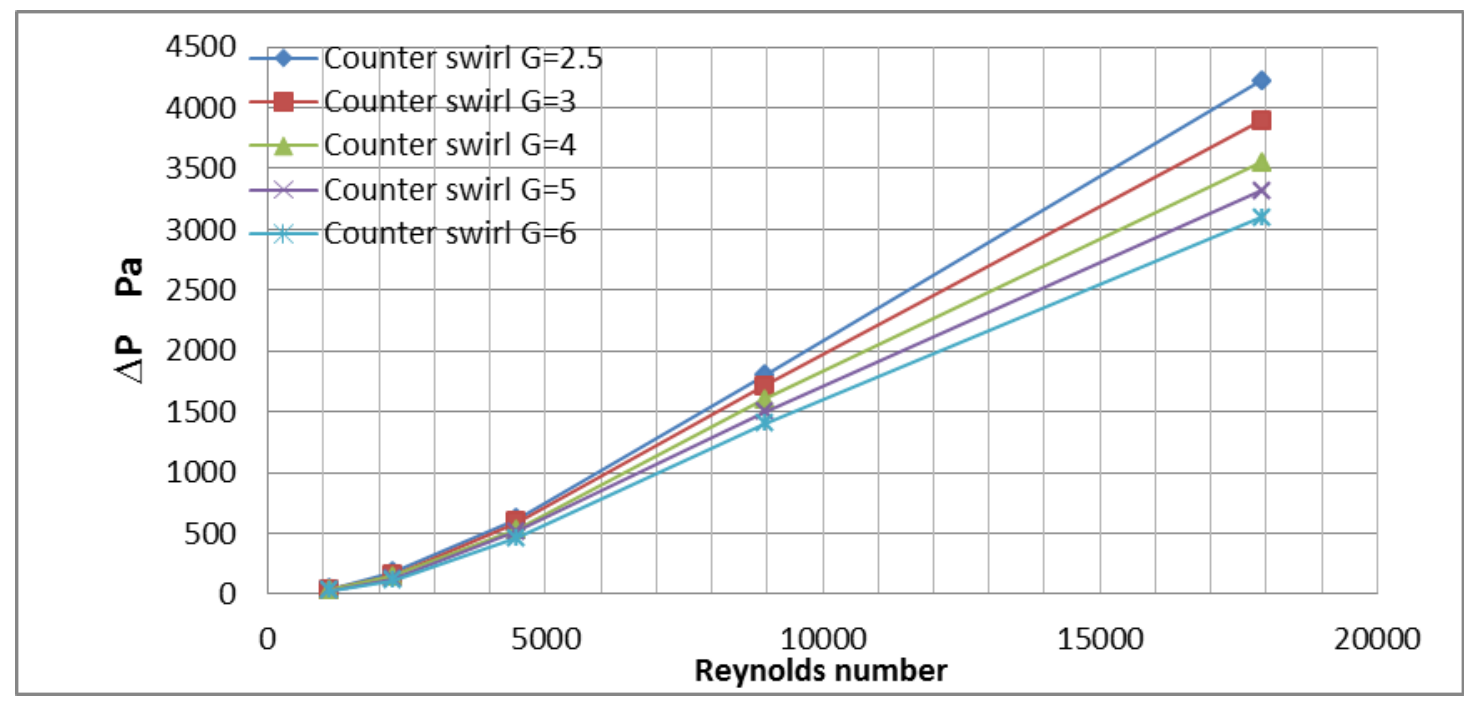

Figure (10): pressure drop for counter swirling tape inserts

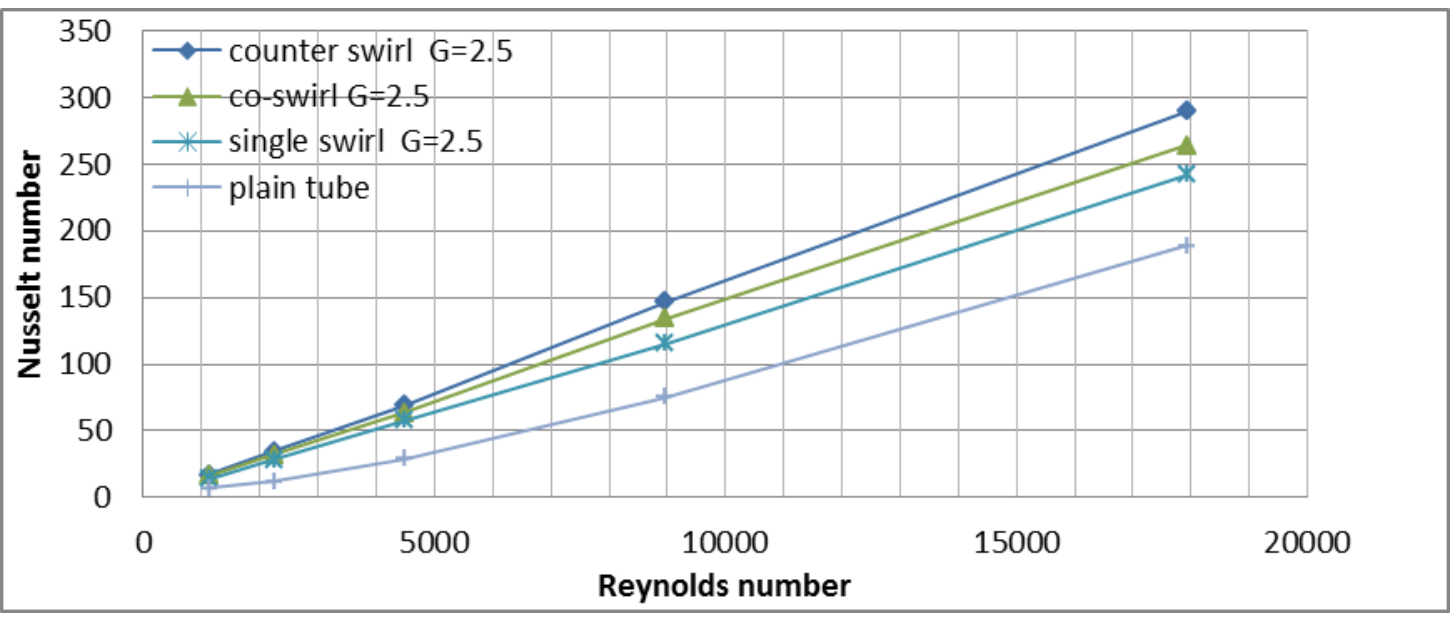

Figure (11): Heat transfer comparison between all insert types. 


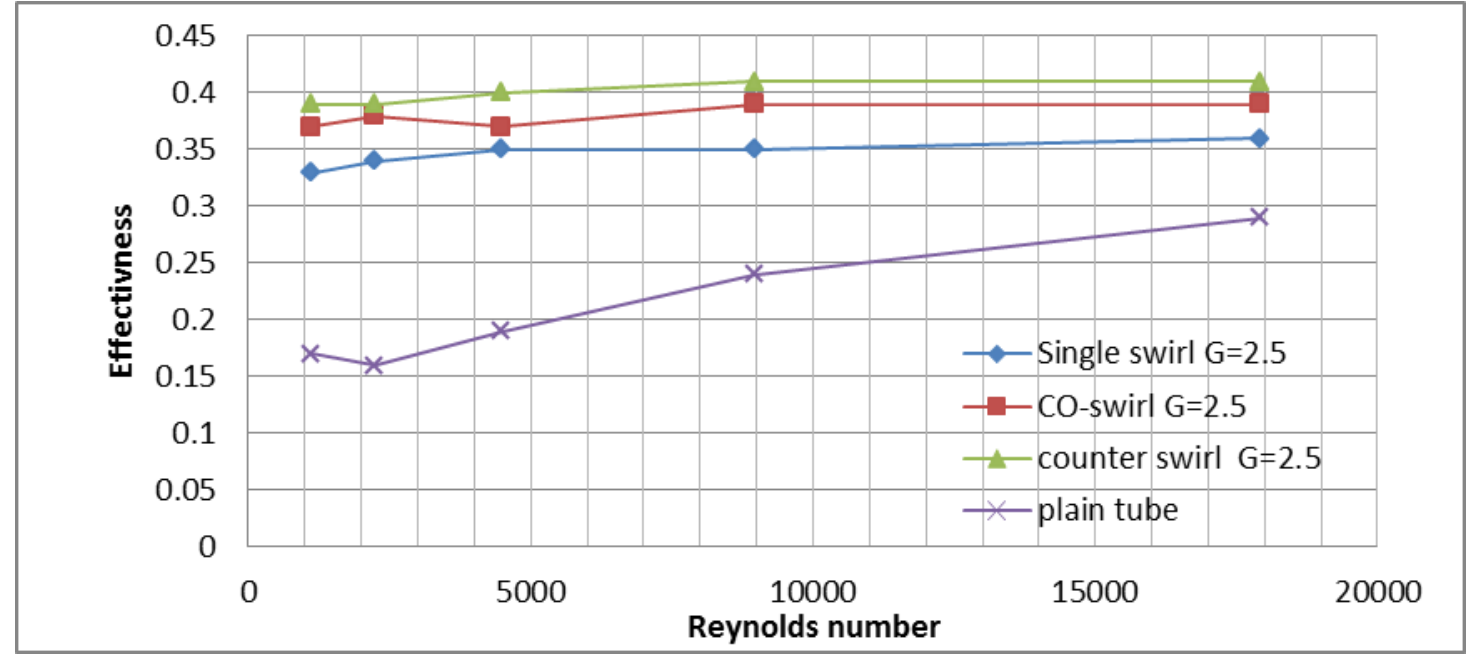

Figure (12): Effectiveness for all inserts and plain tube

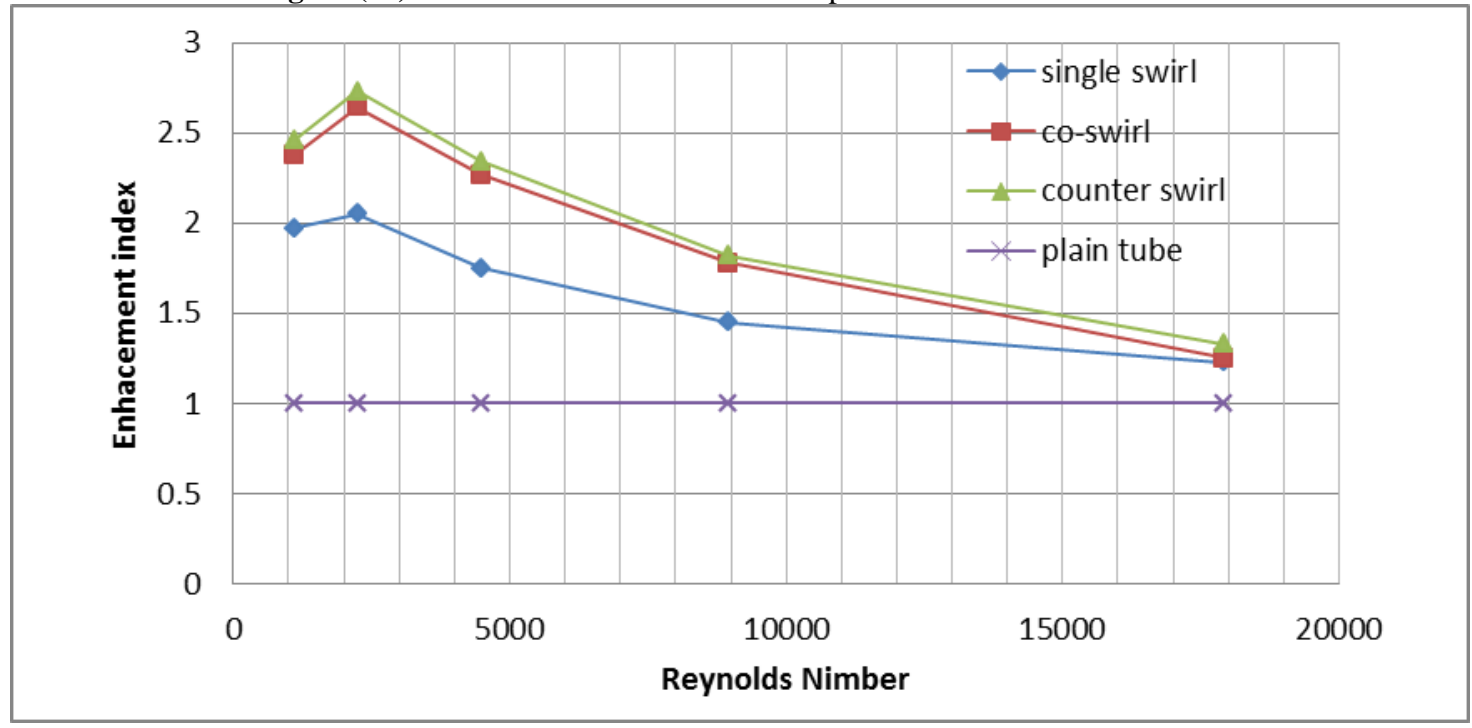

Figure (13): Thermal enhancement index for counter, co-swirling and single tapes inserts.

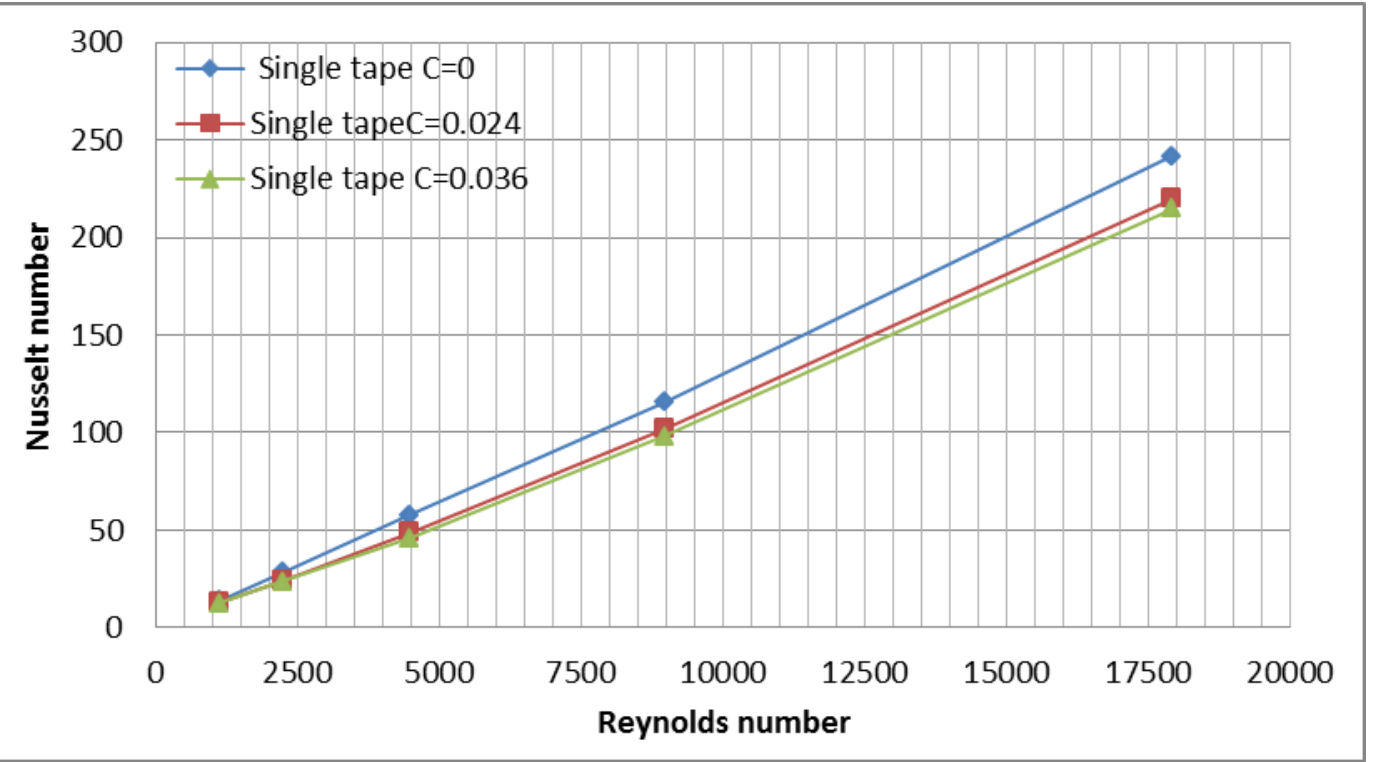

Figure (14): Effect of varying clearance ratio for single tape inserts. 


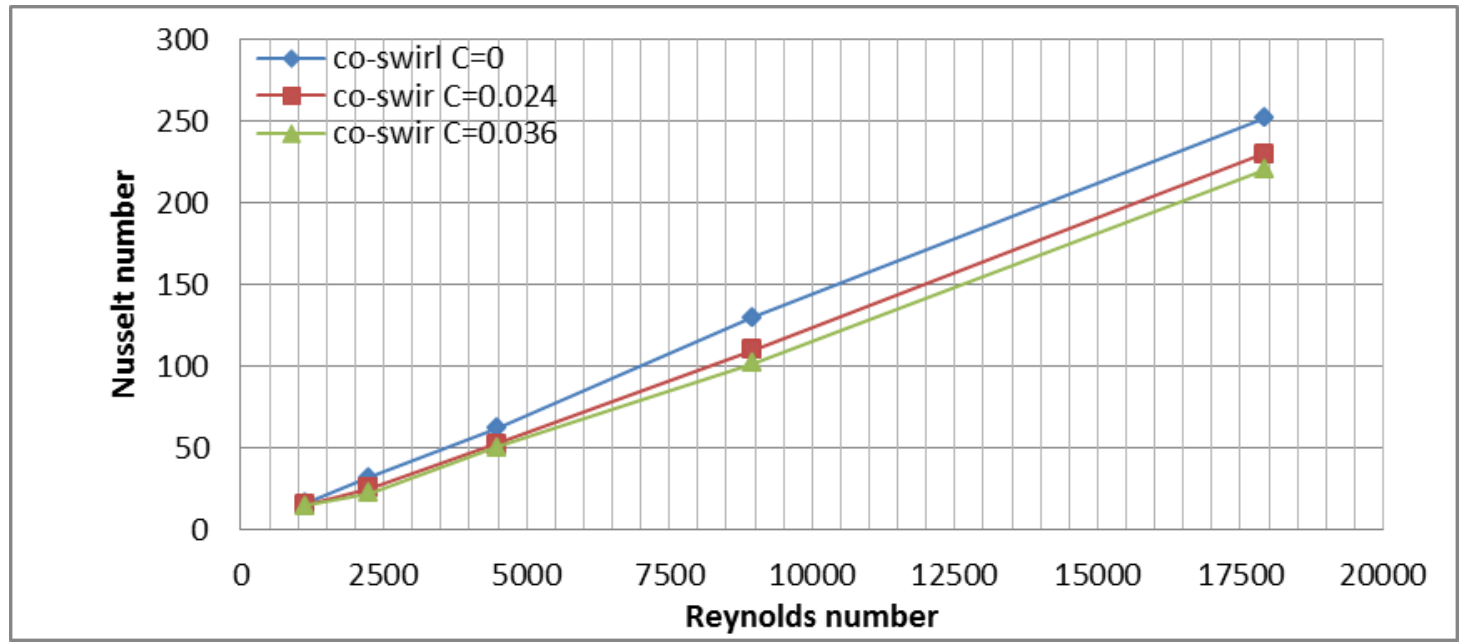

Figure (15): Effect of varying clearance ratio for co-swirling tape inserts.

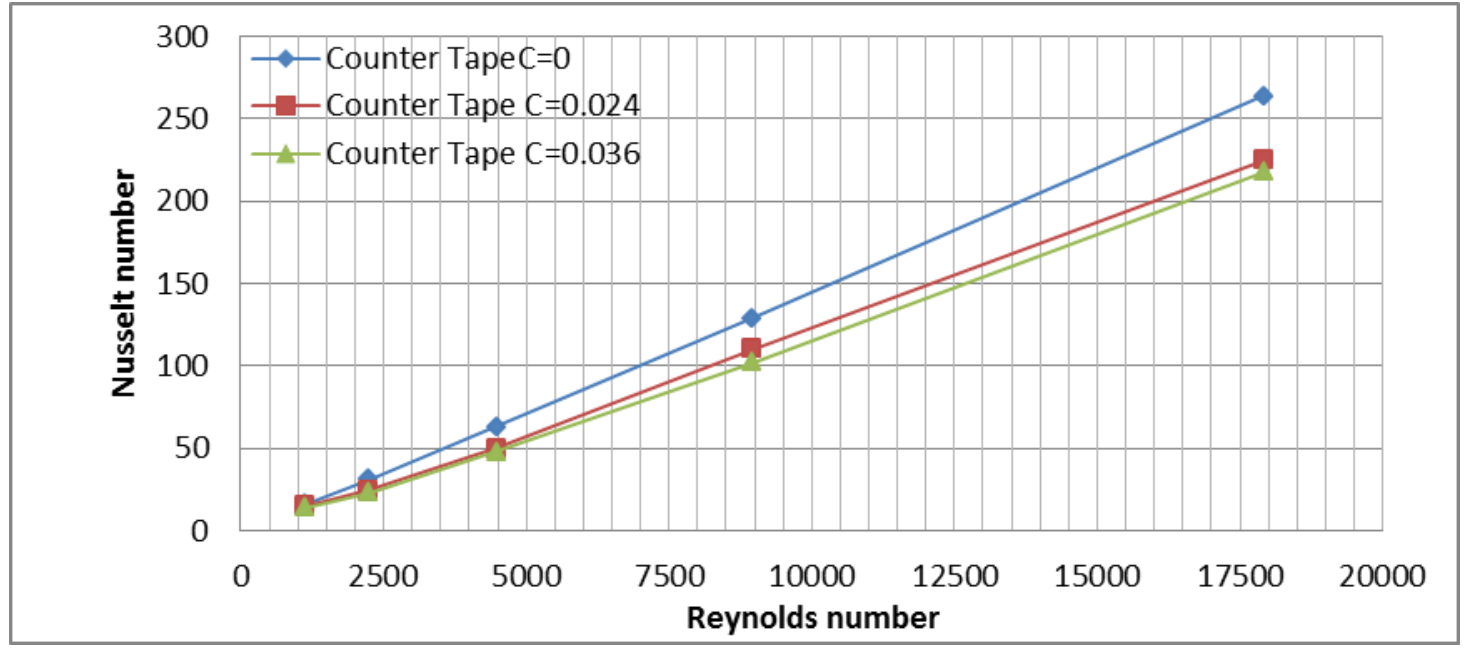

Figure (16): Effect of varying clearance ratio for counter tape inserts.

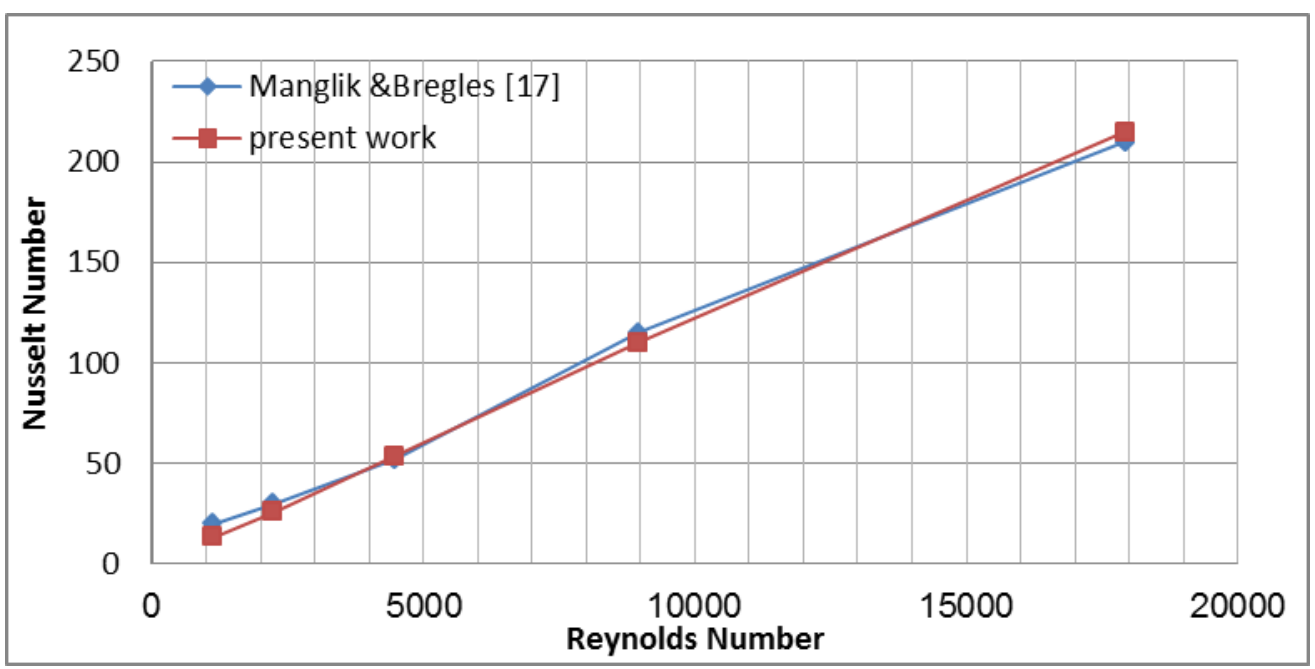

Figure (17): Relation between current work and previous work for single tape insert case 


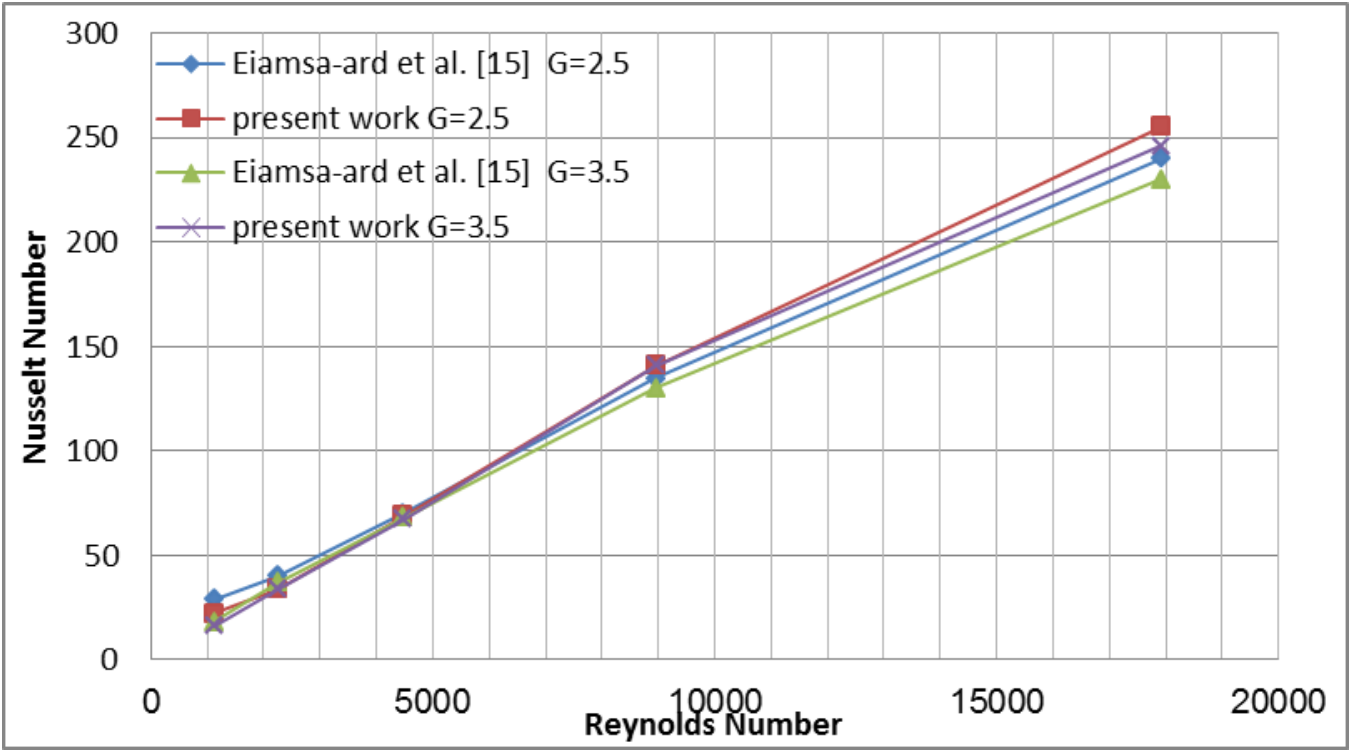

Figure (18): Relation between current work and previous work for co-swirling tapes.

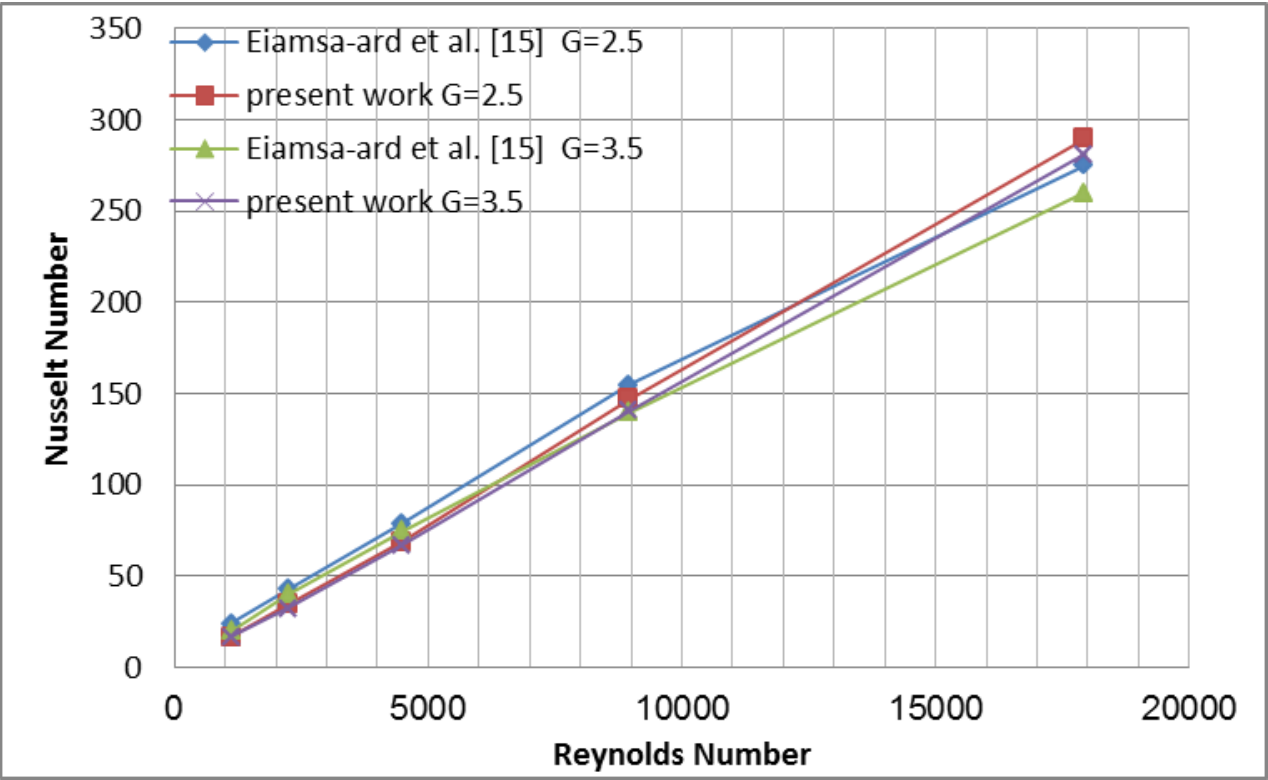

Figure (19): relation between current work and previous work for counter swirling tapes. 

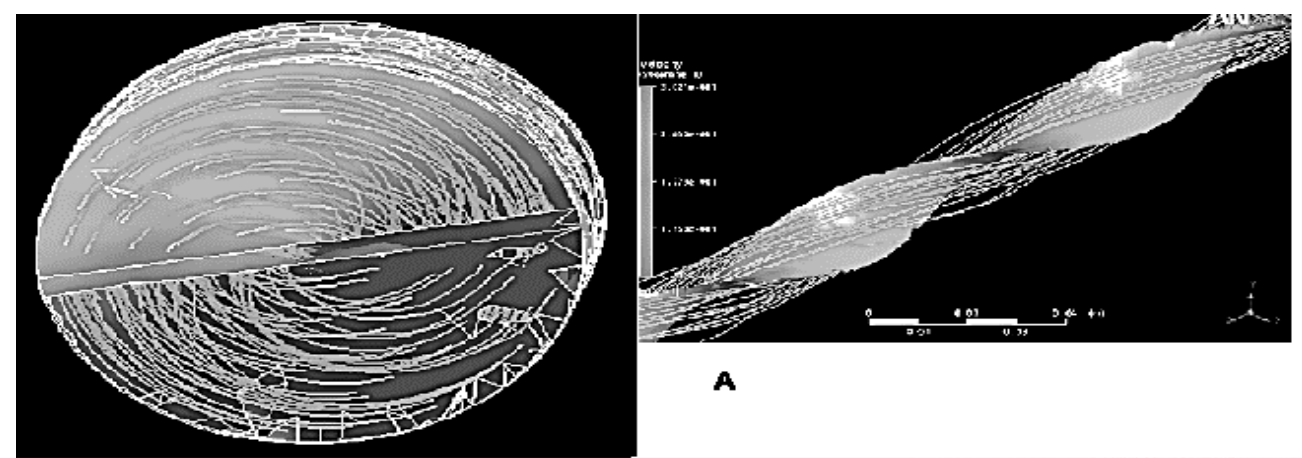

A
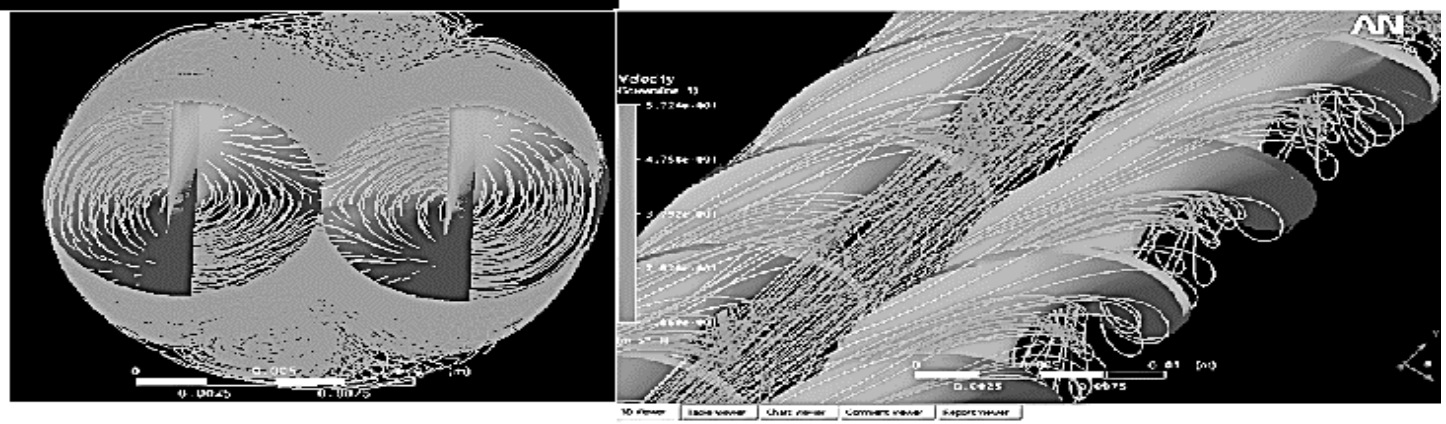

B

Figure20 : Path line flow for A; single tape and B; co-swirling flow.

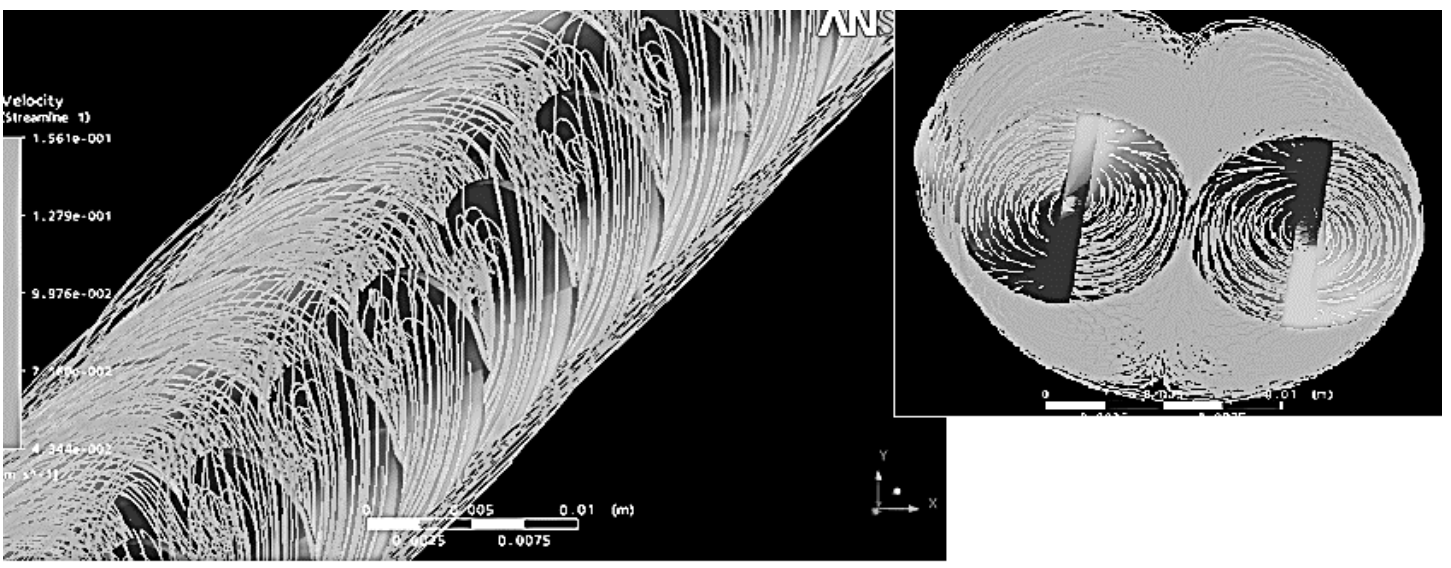

Figure 21: Path line flow for counter tapes 